\title{
Evaluation of Expectation Maximization for the Segmentation of Cervical Cell Nuclei
}

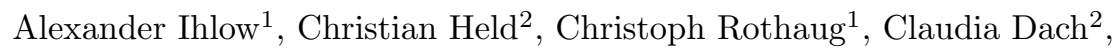 \\ Thomas Wittenberg ${ }^{2}$, Dirk Steckhan ${ }^{2}$ \\ ${ }^{1}$ Ilmenau University of Technology, \\ ${ }^{2}$ Fraunhofer Institute for Integrated Circuits IIS, Erlangen \\ alexander.ihlow@tu-ilmenau.de
}

\begin{abstract}
As cervical cancer is one of the most common cancers worldwide, screening programs have been established. For that task stained slides of cervical cells are visually assessed under a microscope for dysplastic or malignant cells. To support this challenge, image processing methods offer advantages for objective classification. As the cell nuclei carry a high extent visual information, all depicted cell nuclei need to be delineated. Within this work, the expectation maximization (EM) algorithm is evaluated as a yet unused method for this task. The EM was trained on 33 micrographs, where nuclei were manually annotated as reference. The EM was evaluated with varying parameter for the number of classes and with four different color spaces (RGB, Lab, HSV, polar HSV). Segmentation results for all images and parameters were compared to the ground truth, yielding average accuracy and standard deviation for all cells. The best color spaces were RGB and Lab. The best number of classes to be used in the color space was found to be $K=3$. It can be concluded that the EM is an appropriate and useful approach for cell nuclei segmentation, but needs some image post-processing for the elimination of false positives.
\end{abstract}

\section{Introduction}

Since cervical cancer is one of the most common cancers amongst women worldwide, screening programs have been established and are carried out in many countries. For the screening, cervical cells are obtained from the portio vaginalis using a brush during routine examination. The cells are then prepared directly on a slide or are prepared applying a monolayer-preparation (Fig. 1a). To make the cells visible for microscopic examinations, the slide is stained by the method of Papanicolaou, also known as PAP-stain [1].

For visual assessment the stained slides are put under a microscope and screened for dysplastic or malignant cells by a trained specialist. Since this work is tedious and tiring, the viewing and screening process depends on the professional competence as well as the personal and subjective comprehension of the specialist. This comprehension may change during a working day depending on stress, fatigue, and personal issues and may also differ between two people. 
Under these conditions methods of digital image processing can offer advantages for a more objective classification of these highly complex images. Under the assumption of standardized staining and smearing techniques, machines tend to be neutral and immune to inter- and intra-observer changes and influences.

As the nuclei of cervical cells carry a high extent of morphological and textural information, which can be used to diagnose pre-cancerous stages (CIN I-III) as well cancer itself, in a primary step all depicted cell nuclei need to be detected and delineated against the surrounding cell plasma and image back ground.

\subsection{State of the Art}

Various approaches have been suggested to solve the problem of cervical cell nuclei detection and segmentation within automated micrograph analysis. Morphological image processing [2] is well understood and very suitable for objects with well-defined size and form [3,4] and nuclei can be detected using a nucleusshaped structuring element. [4] have suggested the application of a linear tophat-operator [5]. [6] make use of thresholding and morphology, where in a postprocessing step candidates for cell nuclei are selected or rejected using a fuzzy clustering approach. An alternative is the approximation of the nuclei borders by a LoG edge detector [7], which are post-processed using morphological operators for the detection of cell nuclei. Another approach is the circular Hough transform $[4,8]$, which is applied to detect circular structures in gradient images. A technique intended to provide further information about cell nuclei is the use of multi-modal image pairs [9], where an additional fluorescence (FL) staining is applied high lightening only the cell nuclei. On FL image a thresholding operation can be applied to detect the cell nuclei. Disadvantage is that the FL image has to be registered to the PAP brightfield image, which is a difficult and expensive task

Within this work the well-known expectation maximization (EM) approach [10] is evaluated as a yet unused method for automated detection and segmentation of cervical cells.

\section{Materials and Methods}

\subsection{Methods}

Using the EM algorithm, usually a Gaussian mixture model (GMM) is applied to describe the data at hand. The distribution of the annotated color samples $\boldsymbol{x}$ is modeled by a mixture of $K$ classes $f(\boldsymbol{x})=\sum_{k=1}^{K} \pi_{k} f\left(\boldsymbol{x} \mid \lambda_{k}\right)$ with $\sum_{k=1}^{K} \pi_{k}=1$ and $\int_{\boldsymbol{x}} f\left(\boldsymbol{x} \mid \lambda_{k}\right) \mathrm{d} \boldsymbol{x}=1$, where $\pi_{k}$ is the a-priori probability of class $k$ and $\lambda_{k}$ denotes the set of parameters which describes the distribution of class $k$. As a description for the distribution of the mixture components the multivariate Gaussian is chosen due to its convenience. Its equal-probability surfaces describe (hyper)ellipsoids in the $d$-dimensional space. Here, $d=3$ corresponds to the tristimulus color spaces RGB, Lab, HSV, and polar HSV. The model parameters 
$\lambda_{k}$ consist of the mean vector $\mu_{k} \in \mathbb{R}^{d \times 1}$ describing the center of the ellipsoid, and the covariance matrix $\Sigma_{k} \in \mathbb{R}^{d \times d}$ determining its shape and orientation. This yields a GMM of

$$
f\left(\boldsymbol{x} \mid \lambda_{k}=\left\{\mu_{k}, \Sigma_{k}\right\}\right)=\left(1 / \sqrt{(2 \pi)^{d}\left|\Sigma_{k}\right|}\right) \exp \left[-\frac{1}{2}\left(\boldsymbol{x}-\mu_{k}\right)^{T} \Sigma_{k}^{-1}\left(\boldsymbol{x}-\mu_{k}\right)\right]
$$

The EM algorithm [10] is an iterative technique for finding the maximum likelihood parameter estimates when fitting a distribution onto a given data set. During the iterations, the probability $p$ of the $N$ data samples $\boldsymbol{x}_{n}$ belonging to class $k$ is calculated by Bayes' theorem, known as the expectation step $p\left(k \mid \boldsymbol{x}_{n}\right)=\pi_{k} f\left(\boldsymbol{x}_{n} \mid \lambda_{k}\right) / \sum_{j=1}^{K} \pi_{j} f\left(\boldsymbol{x}_{n} \mid \lambda_{j}\right)$ In the subsequent maximization step, updated prior probabilities, mean vectors, and covariance matrices for each class are calculated, using $\pi_{k}^{\text {new }}=X / N, \mu_{k}^{\text {new }}=\frac{1}{X} \sum_{n=1}^{N} Y \boldsymbol{x}_{n}, \Sigma_{k}^{\text {new }}=$ $\frac{1}{X} \sum_{n=1}^{N} Y\left(\boldsymbol{x}_{n}-\mu_{k}^{\text {new }}\right)\left(\boldsymbol{x}_{n}-\mu_{k}^{\text {new }}\right)^{T}$ with $X=\sum_{n=1}^{N} p\left(k \mid \boldsymbol{x}_{n}, \lambda_{k}\right)$ and $Y=$ $p\left(k \mid \boldsymbol{x}_{n}, \lambda_{k}\right)$.

\section{$2.2 \quad$ Experiments}

For the training and evaluation of the proposed methods an image data set of 33 cervical micrographs with a spatial resolution of $1000 \times 700$ pixels has been used where all nuclei were manually annotated as reference or ground truth by an expert. The cells in these probes are typically stained depicting colors from basophile (blue) to eosinophile (red). The images used are ranging from healthy to a dysplastic CIN III state (nearly tumorous), and thus cover the complete range of cervical cells. Fig. 1a shows a typical example of an micrograph with cervical cells, while in Fig. 1b some representative regions of the classes nuclei and rest (including cell plasma and background) were manually marked. Fig. 1c depicts the ground truth of cell nuclei used for later performance evaluation. The EM approach was evaluated with various parameters ( $K=3,4,5,6$ classes $)$ to describe the number of clouds in the color spaces as well as with different color spaces, including RGB, Lab, HSV, and polar HSV. For all experiments, the EM algorithm was terminated after four iterations. In Fig. 2, the segmentation results for the example image from Fig. 1a with the above described parameters is

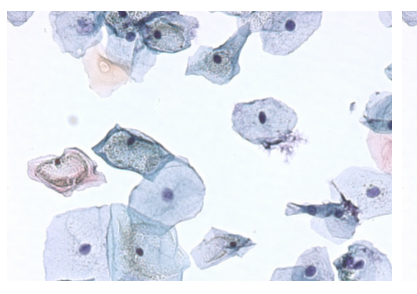

(a)

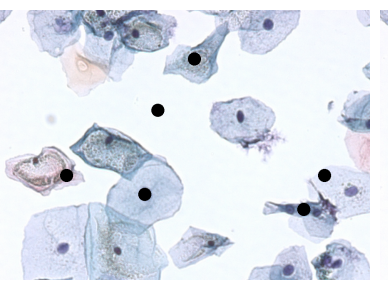

(b)

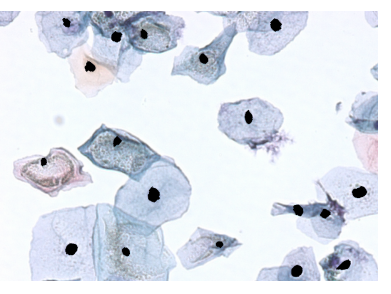

(c)

Fig. 1. Micrograph with cervical cells (a), manual marking of representative areas ( $K=6$ classes) for EM initialization (b), and ground truth of cell nuclei (c). 


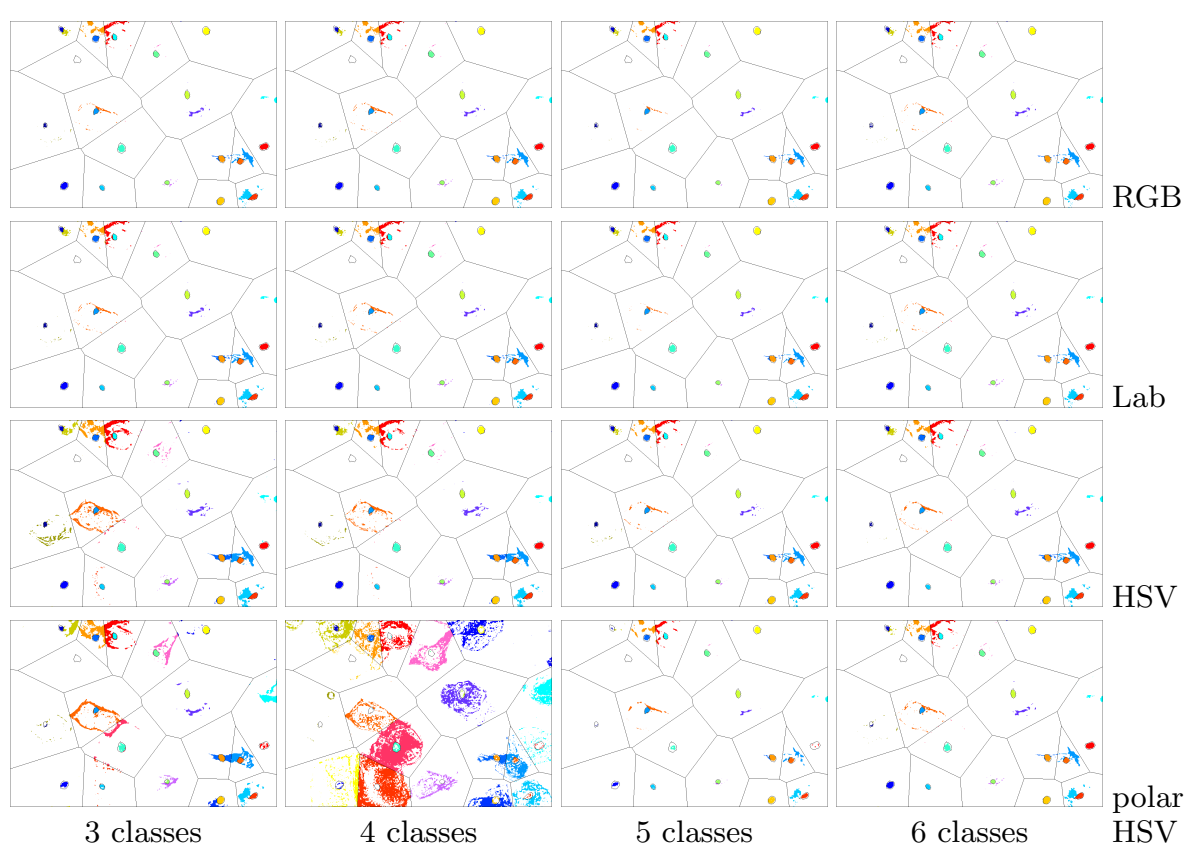

Fig. 2. Segmentation results of micrograph depicted in Fig. 1 for different color spaces and various numbers of classes. Each nucleus is assigned a different color (intensity), false positive pixels are depicted in complementary color (intensity).

depicted. It can be seen that the depending on the two parameters investigated, being the number of classes $K$ and the color spaces, the resulting images show more or less over- and under-segmentation artifacts.

\section{Results}

For evaluation, each image was subdivided into Voronoi regions based on the ground truth nuclei (Fig. 2). The segmentation accuracy $A$ was determined for each Voronoi region by $A=\left(N_{\mathrm{TP}}+N_{\mathrm{TN}}\right) /\left(N_{\mathrm{TP}}+N_{\mathrm{FP}}+N_{\mathrm{FN}}+N_{\mathrm{TN}}\right)$, where $N_{T P}, N_{F P}, N_{T N}$, and $N_{F N}$ denote the number of true and false positive, and true and false negative pixels, respectively. Based on these measurements, the average accuracy and standard deviation of all cells was evaluated as depicted in Fig. 3. It is visible that the RGB and Lab color spaces outperform the HSV and polar HSV color spaces for all $K$. Furthermore, results obtained by EM with $K=3$ in the RGB color space show the best average accuracy values while exhibiting the lowest standard deviation. This is consistent with the example segmentations shown in Fig. 2. Therefore, this parameter set seems to be best suited for the presented task. 
Fig. 3. Average classification accuracy $A$ for the different numbers of classes $K$ and varying color spaces. Error bars indicate standard deviation for the parameterization.

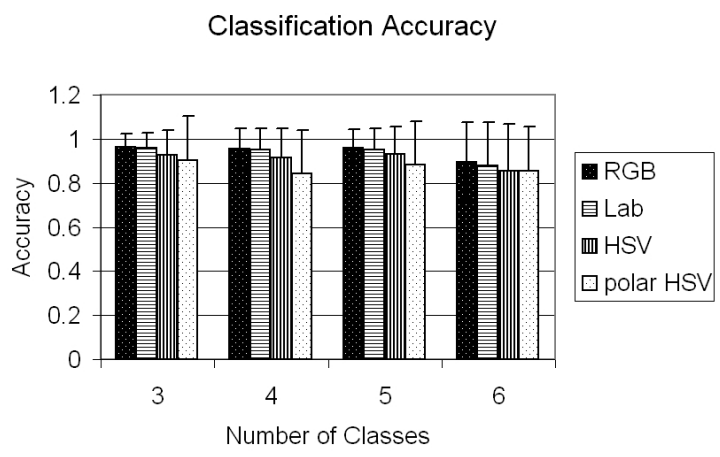

\section{Discussion}

We have presented and evaluated an EM approach using different color spaces (RGB, Lab, HSV, polar HSV) for the segmentation of cervical cell nuclei. Our results indicate that the RGB and $\mathrm{LAB}$ color spaces are most suitable for this task. Using these color spaces the EM is able to perform a segmentation with high sensitivity. A drawback of this method is caused by its strong dependency on the initialization. Future research could focus on the improvement of the specificity of the proposed methods by appropriate post processing steps. Furthermore, some post processing is needed to eliminate false positive pixels.

\section{References}

1. Papanicolaou G. A new procedure for staining vaginal smears. Science. 1942;95:438-9.

2. Soille P. Morphological Image Analysis: Principles \& Applications. Springer; 1999.

3. Anoraganingrum D, Kröner S, Gottfried B. Cell segmentation with adaptive region growing. Proc Int Conf Image Anal Process. 1999; p. 27-9.

4. Köhler H, Wittenberg T, Paulus D. Detection and segmentation of cervical cell nuclei. Proc ICMP \& BMT, Biomed Tech. 2005;50(1):288-9.

5. Abmayer W. Einführung in die digitale Bildverarbeitung. Teubner; 1994.

6. Plissiti M, et al. Automated detection of cell nuclei in PAP stained cervical smear images using fuzzy clustering. In: Proc EMBEC; 2008. p. 637-641.

7. Loukas C, et al. An image analysis-based approach for automated counting of cancer cell nuclei in tissue sections. Cytometry A. 2003;55A:30-42.

8. Thomas A, Davies T, Luxmoore A. The Hough transform for locating cell nuclei. In: IEE Colloq Appl Image Proc in Mass Health Screening; 1992. p. 8/1-8/4.

9. Lindblad J, Bengsston E, Wählby C. Robust cell image segmentation. Pattern Recogn Image Anal. 2004;13(2):157-167.

10. Dempster A, Laird N, Rubin D. Maximum Likelihood from Incomplete Data via the EM algorithm. J Royal Stat Soc B. 1977;39(1):1-38. 